\title{
Smart Sensors and Small Robots
}

\author{
Mel Siegel \\ Robotics Institute \\ Carnegie Mellon University \\ Pittsburgh, PA 15213-2605, USA \\ Phone: +1 412268 88025, Fax: +1 4122685569 \\ Email: mws@cmu.edu, URL: http://www.cs.cmu.edu/ mws
}

\begin{abstract}
Robots are machines that sense, think, act and (more recently) communicate. Sensing, thinking, and communicating have all been greatly miniaturized as a consequence of continuous progress in integrated circuit design and manufacturing technology. More recent progress in MEMS (micro electro mechanical systems) now holds out promise for comparable miniaturization of the heretofore-retarded third member of the sense-think-actcommunicate quartet. More speculatively, initial experiments toward microbiological approaches to robotics suggest possibilities for even further miniaturization, perhaps eventually extending down to the molecular level. Alongside smart sensing for robots, with miniaturization of the electromechanical components, i.e., the machinery of robotic manipulation and mobility, we quite naturally acquire the capability of smart sensing by robots. This paper summarizes the state-of-the-art, particularly with respect to the role of and the requirements for sensing. Three main cases are described: (1) the robot's mission is to carry sensors; (2) environmental sensing is required for the robot to accomplish its mission; and (3) proprioception, i.e., sensing for robot self-awareness.
\end{abstract}

Keywords - autonomous, mobile, robot, nanorobot, microrobot, minirobot, sensing, remote sensing, teleoperation, proprioception

\section{INTRODUCTION}

When we were inventing robotics as an academic discipline, we divided the field into specialties that correspond to Allen Newell's three-component definition: a robot is a machine that senses, thinks, and acts [1]. The robotics educational curriculum that emerged correspondingly requires each student to become proficient in the sciences and technologies of perception, cognition, and manipulation and mobility.

Sensing: Robots require sensors for many reasons, most of them falling into one of three broad categories: (1) sensing is the purpose of the mission, e.g., to gather and transmit images and chemical analyses of rocks on a planetary exploration mission ("robots for sensing" types of applications); (2) sensing is required to enable the machines to survive in the mission's environment, e.g., to recognize the obstacles between present position and desired position ("sensing for robots" types of applications); and (3) proprioception, enabling the robots to sense their own "personal" configurations, and their relationships to the environment, e.g., to achieve a pose that permits the manipulator to lift an environmental object in a manner that is consistent with its own materials strength and power limitations ("sensing for robotics" types of applications). It is perhaps already apparent that the really interesting applications also involve mobility and communication.
Thinking: Cognition is a prerequisite for adaptability, without which no real machine could operate for any significant time in any real environment. No predetermined plan could realistically anticipate every feature of the environment - and if it could, the mission might be utilitarian, but it would hardly be interesting, as what would be its purpose if there were no new knowledge to be gained? Anyway, even a toy mission in a completely known environment would soon fail in open-loop operation, as accumulated mechanical and sensor errors would inevitably lead to disastrous discrepancies between predicted and actual states. Of course, "dumb" "black-box" mechanisms do sometimes exhibit seemingly intelligent behavior, but only in very limited domains [2].

Acting: An ability to act on the environment is regarded, alongside an ability to sense it, as an essential requirement for the evolution of intelligence. Although both manipulation and mobility are necessary components of action, we can imagine missions - and we can identify many real missions in which either no manipulation is required, or in which very simple manipulation that is actuated by the mobility component is adequate. Thus we focus mostly on mobility. In robotics' beginnings, mobility was more a dream than a reality: robots were then essentially one-armed iron-laborers bolted at their waists to the factory floor. But much progress has been made, particularly in the mobility domain vs. the manipulation domain. The current image of a robot, both in the research community and in the public's eye, is of a rather agile mobile creature, propelled usually by wheels - though sometimes by legs - usually in a two-dimensional environment, but sometimes by aerodynamic lift or rocket propulsion in a three-dimensional environment.

Communicating: As mobility becomes more-and-more a taken-for-granted attribute of the machines we call robots, it seems inevitable that we will have to append communicate to the original sense, think, and act list. Only with effective communication will mobile robots be able to cooperate effectively with each other, and with the humans they are meant to serve [3].

In this "state-of-the-art review" I will, within the sense-thinkact-communicate framework, summarize the current historical moment for small mobile robots. I will particularly em- 
phasize, for the instrumentation-and-measurements-oriented audience, the sensing component - where it is strong, where it is weak, and where it is absent. In the main text and in the conclusions, I will, as comprehensively as I can, summarize and critique the state-of-the-art, and as best I can, I will offer educated guesses about the near term prospects, long term promises, and the scientific and technical gaps that will need to be bridged in order to achieve the prospects and promises.

Section II briefly summarizes the BACKGROUND, particularly with respect to the driving forces underlying the requirements for small sensory robots, and some of the inherent bottlenecks that will need to be overcome to achieve practical success. Section III is a lighthearted census of the SIZEDISTRIBUTION OF ROBOTS, including an attempt to discern from the compiled statistics how terms like nanorobot, microrobot, etc., are defined by common usage, and the identification of a scaling law for deducing the appropriate SI-prefix to put in front of "robot" as a function of its characteristic linear dimension. Section IV discusses SMALL ROBOTS in four subsections corresponding to the categories molecular robots, nanorobots, microrobots, and millirobots (or minirobots). Section V constitutes the CONCLUSIONS, structured as a systematic summary the state-of-the-art, the future requirements, and the future prospects for sensing and 'smart sensing' for the small robots discussed in the previous section.

\section{BACKGROUND}

With mobility taken as a given, both individual robot size and the number of robots constituting a system become crucial issues. If we are to be adequately served by mobile robots, we will need large numbers of them. If we are to have large numbers, they will have to be cheap; and to be cheap - and also to be able to work effectively in the environments wherein most applications are anticipated - they will have to be small ${ }^{1}$. Typical anticipated applications, to name a few, include surveillance, inspection, and exploration and repair inside more-or-less sealed systems, from aircraft fuel lines and tanks to the conduits and cavities of the human body.

Early mobile robots were behemoths relative to their modest capabilities. Sensing, thinking, and communicating hardware have evolved rapidly from macro- to micro-scale; they now seriously promise to reach even the molecular scale. But actuation - mobility and manipulation - have made much less progress toward miniaturization. Despite impressive and important demonstrations of progress-in-principle towards MEMS devices, actuation for real-world manipulation and

\footnotetext{
${ }^{1}$ In the question-and-answer session following a 1985 lecture at Carnegie Mellon University, Akio Morita, founder of the Sony Corporation, was asked why he is so passionate about making his company's products ever smaller [4]. His disarmingly simple answer was - surprisingly - a surprise to most of the audience: smaller uses less material, so it is cheaper.
}

mobility applications resists great shrinkage in practice. Even the MEMS approach has to date produced only largeinsect sized robot-like artifacts, and these remain laboratory curiosities, despite the numerous practical medical, industrial, etc., applications that beg for these and even smaller devices.

Furthermore, whatever progress is made toward smaller actuators, there looms an inevitable barrier: the limited energy carrying capacity of small devices. For a machine with characteristic linear dimension $\mathrm{R}$, energy-carrying capacity scales as $\mathrm{R}^{3}$, whereas power dissipation to internal and external friction scales more-or-less as $\mathrm{R}^{2}$. Thus the operating time of any stored-power device is more-or-less proportional to $\mathrm{R}$. In practice, the best self-powered small robots built to date have about the same operating time as the "best" microbes have between feedings: less than one hour. But the microbes are 10,000 or so times smaller than the smallest actual robots, which means, according to this scaling relation, that their energy carrying capacity is more efficient by the same factor. The size of this gap forcefully argues that, for a long time into the future, small robots will have to be powered either by a "feeding" mechanism that extracts energy from the environment, or through an umbilicus.

Most readers will recognize that an umbilicus is the Achilles heel of current generation full-sized mobile robots: these machines most typically fail by tangling or cutting their own umbilicus - it is the robotic analog of "tripping over your own shoelaces". Until we learn - perhaps from future advances in biological science - how to make tiny robots that can forage for their energy, small mobile robots will require some kind of umbilicus to accomplish any task that requires more than a few minutes. Given the many-times-demonstrated impracticality of even the most thoughtfully designed physical umbilicus, only a completely ethereal umbilicus, e.g., a tracking laser beam with sufficient power to "feed" the robot, seems to be a viable alternative. With an ethereal umbilicus, being small is actually highly advantageous: surface-to-volume ratio increases as $\mathrm{R}^{-1}$, so the requirements for both total transmitted power and beam power density both decrease substantially as the robot becomes smaller.

With respect to sensing, we caution the reader that whereas the requirements are well known, and, for most of them, micro- if not nano-scale sensors have been demonstrated and, in some cases, are already available off-the-shelf, little has yet been done in the way of sensor integration with any but the largest of the small robots we will discuss. The critical reader should keep these sobering BACKGROUND considerations in mind when reading the following section on the SIZE DISTRIBUTION OF ROBOTS, and the subsequent (more serious) sections that summarize realizations of SMALL ROBOTS and the SENSING AND SMART SENSING issues involved in implementing and applying them. 


\section{SIZE DISTRIBUTION OF ROBOTS}

Recent web searches for \{SI-prefix $\}$-robot [5] yielded 1.27 $10^{6}$ hits for robot, 8 for millirobot plus another 408 for minirobot $^{2}, 1380$ for microrobot, 324 for nanorobot, and a few bogus hits - not related to actual robotics research - for exarobot, terarobot, gigarobot, and megarobot. The distribution is shown on a log-log plot in Figure 1. Regrettably, unlike the internationally standard SI units "meter", "volt", etc., there is no standard definition for one "robot". Nor do the usual SI prefixes commonly employed to describe robot sizes imply strict relative scaling by successive factors of $10^{3}$. So, for the purposes of this review, I will define terminology in terms of characteristic linear dimension as follows:

$$
\begin{aligned}
& \text { - "robot": } \sim 1 \mathrm{~meter} \\
& \text { - "millirobot": } \sim 10 \mathrm{~cm} \\
& \text { - "microrobot": } \sim 1 \mathrm{~mm} \\
& \text { "nanorobot": } \sim 10 \mu \mathrm{m}
\end{aligned}
$$

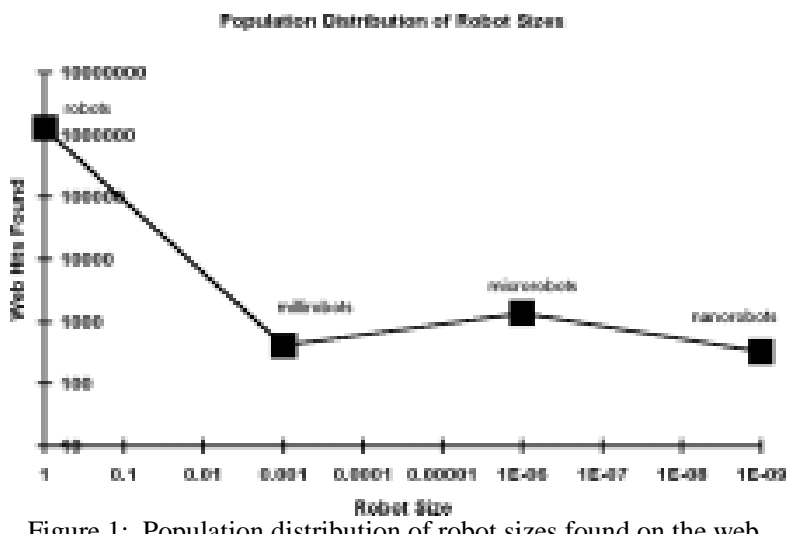

Figure 1: Population distribution of robot sizes found on the web.

Thus the characteristic linear dimension of a robot changes by a factor of 100 for each prefix change of 1000 , i.e., it seems that, by popular usage, the characteristic linear dimension of a robot is proportion to the 2/3-power of its SI prefix.

Figure 2 shows an example of a robot, a millirobot, a microrobot, and an example of activity at the nanorobot scale.

At the top left of Figure 2 we see CIMP, an aircraft skin inspection robot built the author's group at CMU [6]. CIMP's characteristic dimension (height) is about $1 \mathrm{~m}$, i.e., CIMP is "person-sized". Made of lightweight materials (mostly kraft paper honeycomb and plastics), CIMP can be carried with difficulty by one person, and easily by two. Primary sensors are a high-quality close-up stereoscopic camera for inspection and a wide-angle stereoscopic camera for navigation.

At the top right of Figure 2 we see the Palm Pilot "millirobot" [7] developed by my CMU colleague Illah Nourbakhsh's group. It is essentially a Palm Pilot PDA with outboard wheels. Its diameter is about $20 \mathrm{~cm}$. All the parts needed to build a Palm Pilot Robot can be purchased in kit form, and

\footnotetext{
${ }^{2}$ Since "milli" and "miNi" differ by only a backslash (\) we consider the two terms equivalent.
}

software source code can be downloaded via Nourbakhsh's website referenced above. The Palm Pilot Robot's sensors include infrared ranging devices and a fluxgate compass.

At the bottom left of Figure 2 we see two members of the Allumette (match) "microrobot" [8] family developed at the ETHZ (Swiss Federal Institute of Technology - Zurich) Institute of Robotics. Unfortunately, the Allumette family's sensor complement (if any) is not discussed.

At the bottom right of Figure 2 we see the letters "USC" spelled out in $15 \mu \mathrm{m}$ gold balls that were manipulated by a scanning probe microscope (SPM) at the University of Southern California (Los Angeles) Laboratory for Molecular Robotics. The authors describe the manipulator as a "nanorobot" [9]. The referenced article describes a client/server architecture whereby remote users can operate the instrument in a sense, think, act (and, although unstated, communicate) cycle. A similarly named and implemented effort is independently underway at ETHZ [10], and probably other places.
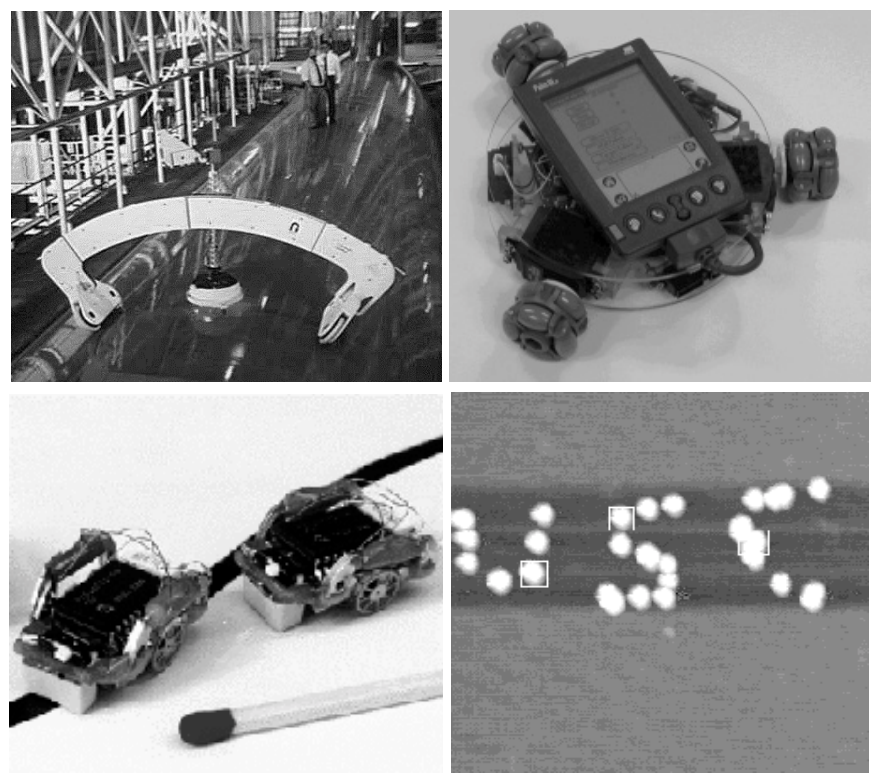

Figure 2: (top left) CIMP aircraft inspection robot, height $\sim 1 \mathrm{~m}$. (top right) Palm Pilot "millirobot", diameter $\sim 20 \mathrm{~cm}$ (bottom left) Allumette "microrobot", dimensions $\sim 10 \mathrm{~mm}$.

(bottom right) "USC" written in $15 \mu \mathrm{m}$ diameter gold balls positioned by a scanning probe microscope (SPM) so-called "nanorobot".

In this review I will consider milli- and smaller- robots. Below the above-defined nanorobot scale, some researchers are already considering robotic devices and systems at microbial and molecular scales. The following section, SMALL ROBOTS, begins at the small end of the scale, molecular robots, and proceeds through nanorobots, microrobots, and millirobots (or minirobots). This progression moves from almost completely speculative work, through a regime in which a little groundwork has been laid, then into areas where partly functional prototypes have been built, and into an where area experimental platforms can be purchased. 


\section{SMALL ROBOTS}

\section{Molecular Robots: the smallest of the small}

Let's begin with the smallest of the small: Ron Weiss, a $\mathrm{PhD}$ candidate at the MIT AI lab, is studying how to harness microbes for sensing, actuating, computing, and communicating [11]. If successful, this would make the microbe colony according to the accepted definition - a robot.

Weiss points out that microbes sense their environment, process the sensory information, and act appropriately in response. He also notes that they are energy efficient, selfreproducing, and - of course - small, all of these making them attractive candidates for use as computational elements. His approach would add "biochemical logic circuitry" to control microbial intra- and inter-cellular processes.

He uses recombinant DNA-binding proteins to represent signals (communication), and recombinant genes to "compute" (think) by regulating protein expression. His experiments with Escherichia coli implement the AND, NOT, and IMPLIES logic operations via "biochemical gates", and he has prototyped several small circuits that combine these elements. To support his work, Weiss has prototyped a genetic circuit simulation and verification tool (BioSpice), and a programming language (Microbial Colony Language, MCL). He is exploring the classic biologically-inspired computing issue of how to achieve globally reliable behavior from a large number of individually unreliable computing elements that communicate only locally.

Weiss foresees applications in drug and biomaterial manufacturing, delivery, and action, intelligent materials, microsensors, microactuators, and micromanufacturing. These may be only a small subset of the opportunities. For example, we can see prospects for medical applications such as the repair or even the regeneration of muscle, bone, and organ structures, and for manufacturing applications in which large structures, e.g., automobiles or even buildings, could be grown from a tiny seed supplied with appropriate nutrients and energy.

\section{Nanorobots: there's room at the bottom!}

Late in 1959, Feynmann presented a talk at the annual meeting of the American Physical Society entitled "There's Plenty of Room at the Bottom" [12]. It is often cited as the birth nanotechnology. It ends with two $\$ 1000$ challenges that Feynmann says he expects will be collected in a very short time. The first challenge is to "take the information on the page of a book and put it on an area 1/25,000 smaller in linear scale in such manner that it can be read by an electron microscope". The second is to make "a rotating electric motor which can be controlled from the outside and, not counting the lead-in wires, is only $1 / 64$ inch [0.4 mm] cube". For the first challenge, if we accept digital storage on magnetic stor- age media, a typical page has about 4000 characters on about $500 \mathrm{~cm}^{2}$, or about 8 bytes $/ \mathrm{cm}^{2}$. Increasing this area density by a factor of $25,000^{2}$ requires $5 \mathrm{~GB} / \mathrm{cm}^{2}$. Current off-theshelf magnetic disks fail to achieve this density by a factor of about 100. If we insist that the challenge requires us to store an image of the page and not just its text representation, then the challenge fails, even with lossy compression, by a factor of about 10,000 . The second challenge calls for a rotating electric motor in a $(0.4 \mathrm{~mm})^{3}$ volume. The smallest off-theshelf rotating electric motors available seem to be about 2 $\mathrm{mm}$ diameter by $6 \mathrm{~mm}$ length [13], which falls short of Feynmann's challenge by a factor of about 400 . However silicon micromachined gear trains, lever-actuators, etc. (see Figure 3 ), have actually been built within a volume well under that specified in the challenge, and the prospects for realizing a true motor by this technology appear sanguine.
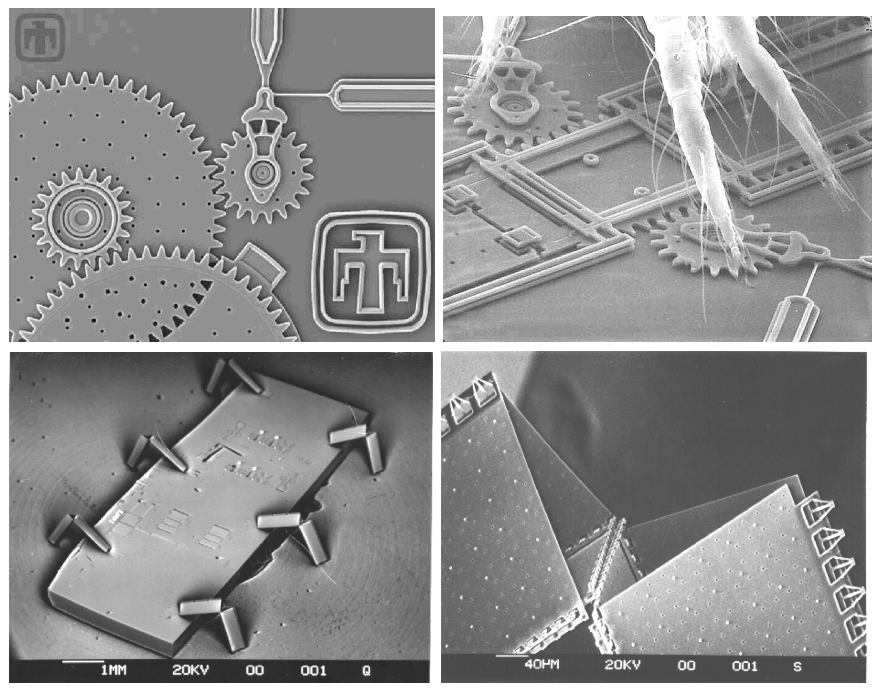

Figure 3: (top left) Sandia gear train, typical of MEMS micromotor parts.[14] (top right) Sandia gear train, with spider mite leg for size comparison.[15] (bottom left) Berkeley silicon bug prototype walking robot.[16]

(bottom right) Detail of elbow joint hinge on previous silicon bug.[16]

Nanorobotic devices and components of the sort illustrated in Figure 3 are "demonstrations of principle", not working systems. The necessary structural components and some apparently viable microactuators have been separately demonstrated, but, to the best of the author's knowledge, none of these structures has yet been integrated with even rudimentary sensing, thinking, acting, or communicating machinery.

\section{Microrobots: watchmakers making robots}

Microrobots, as we have defined them, have characteristic dimensions in the millimeter regime. They can be - and usually are - built using "ordinary" small component manufacturing technology vs. microfabrication, i.e., they don't require integrated circuit manufacturing technology. Microrobot components are often entirely off-the-shelf. Figure 4 shows an example from the group of Doug Adler and Ed Heller of Sandia National Laboratories (New Mexico) [17]. This par- 
ticular robot, apparently unnamed, has a body made by stereolithography, but other parts are off-the-shelf, with the exception of the microprocessor, which is built up from a purchased die mounted on a glass substrate using Sandia's inhouse facility. Its volume is about $4 \mathrm{~cm}^{3}$, or about $16 \mathrm{~mm}$ in each dimension. Its weight and volume are dominated by the power supply, three button cells, which carry enough energy for the robot to operate for 15-20 minutes untethered. Except for one temperature sensor, it is "unsensored".

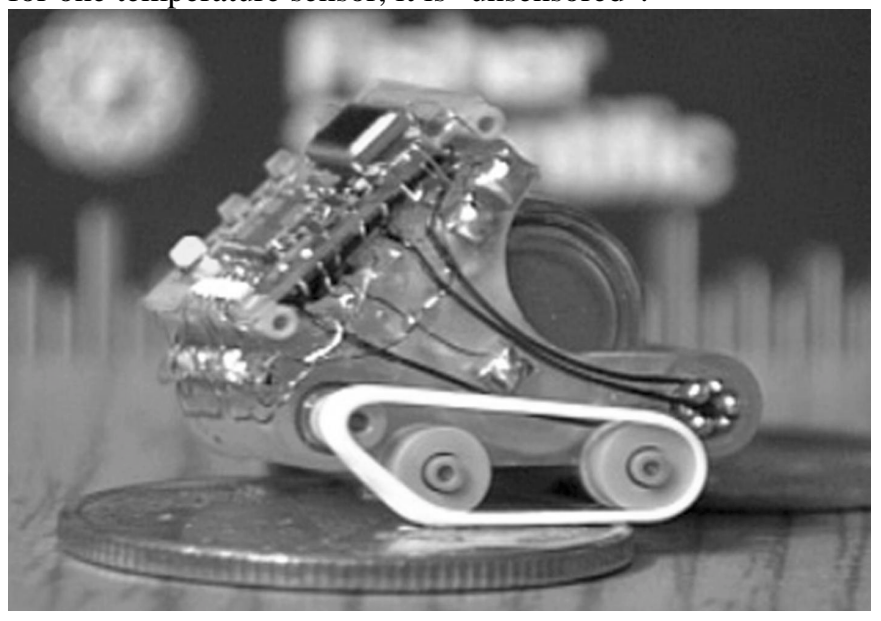

Figure 4: Sandia's toy-balloon treaded Microrobot. The out-of-focus scale in the background is in units of $1 / 16$ inch, equal to $1.5875 \mathrm{~mm}$. [18]

An earlier, slightly larger model of this microrobot $(40 \times 19 \times$ $18 \mathrm{~mm}$, about $14 \mathrm{~cm}^{3}$ ), called MARV (Mini Autonomous Robot Vehicle) [18], had commercial obstacle detecting sensor, radio, and temperature sensors, and two in-housedeveloped RF sensors that steer the robot on a path centered on a (potentially) buried RF-carrying wire. Both versions are controlled by a small on-board microprocessor with $8 \mathrm{~K}$ of memory. Sandia a (US "national security" lab) foresees applications using of swarms of miniature autonomous vehicles for locating and disabling land mines, detecting chemical and biological weapons, verifying treaties, etc.

Several other groups have described similar microrobots. A notable one is the Autonomous Systems Laboratory at Ecole Polytechnique Federale de Lausanne (EPFL), whose "Alice" family members number several dozen [19]. Alice 99 [20] has a 10 pin "extension connector" which accepts either a sensor module or and radio communication module. The sensor module contains four proximity sensors (SFH-900), a four channel ADC, an 8 channel MUX, a serial port interface, and an IR link for inter-robot communication in multi-robot application scenarios, or for manual teleoperation via an infrared remote control for a television set. The radio communication module provides a range of approximately $10 \mathrm{~m}$ for both robot-robot and robot-PC communications.

Thus in contrast to the promise of nanorobots, we have the reality of microrobots: complete self-contained working systems that sense, think, act, and communicate, albeit simply.

\section{Millirobots (or Minirobots): is it smaller than a breadbox?}

As illustrated by the small sampling shown in Figure 4, robots with a characteristic linear scale of $10-20 \mathrm{~cm}$ are experiencing a veritable population explosion. These millirobots (or minirobots), substantially smaller than a breadbox but substantially bigger than a breadcrumb, encompass by fare the largest subpopulation of small robots. Some of them are essentially educational toys, of varying sophistication, e.g., the BoeBot [21], the Runabout [22], and the Descartes [23] examples at the top left, middle left, and middle right. Others are meant to be platforms for serious robotics research, both on mechanisms and on algorithms, e.g., the EPFL Khepera modular platform [24] at the top right. Still others are serious prototypes of operational designs, like the SAIC SuBot [25] "spherical" military reconnaissance robot at bottom left and the Helsinki University of Technology Automation Technology Lab's SubMar [26] autonomous water-sampling robot at bottom right.
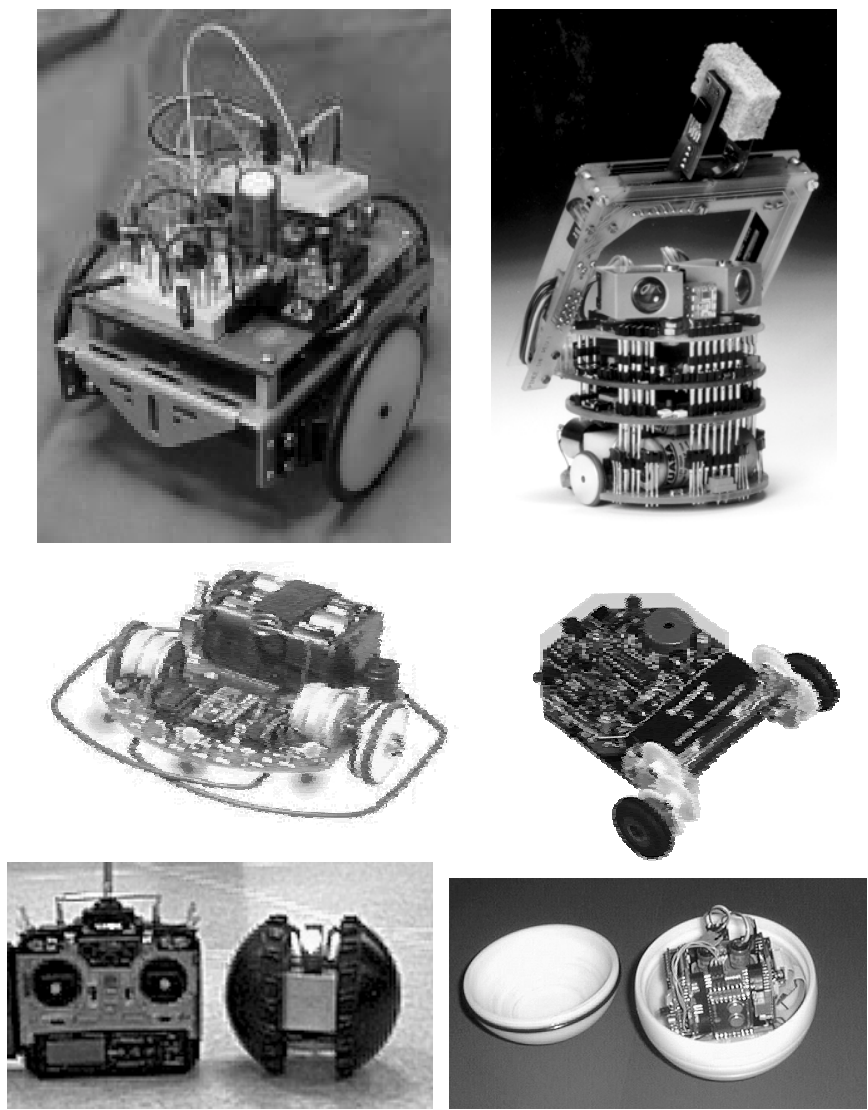

Figure 4: (top left) BoeBot, approximately $10 \mathrm{~cm}$ linear dimension [21]. (top right) Khepera, $5.5 \mathrm{~cm}$ diameter, holding a sugar cube [24]. (middle left) Descartes, $15 \mathrm{~cm}$ diameter [23].

(middle right) Runabout, approximately $12 \mathrm{~cm}$ linear dimension [22] (bottom left) SuBot 16, cm "spherical", military reconnaissance robot [25]. (bottom right) SubMar, $11 \mathrm{~cm}$ diameter underwater sampling robot [26].

While many of the "minirobot" class are only about as sophisticated as some radio-controlled toys, others, like the Khepera and the SubMar, are sensor-intensive, autonomous, and about as intelligent as state-of-the-art full-sized robots. 


\section{CONCLUSIONS}

By now the reader must be wondering whether the title is some sort of fraud: the review is called "Smart Sensing and Small Robots", but two-thirds of it is about robots, not about sensors, many of the robots described are still little more than dreams, and most of the realized robots described, lacking onboard intelligence, lacking smart sensors, and lacking sophisticated communication, fail to meet the definition of robots! Well, this is a "state-of-the-art" review, and this is the state-of-the-art! So let us look to the future and ask:

- What sensors are needed on a (small) robot?

- Are they available - even if not small??

- What are the prospects for making them small?

- Do they need to be 'smart sensors'?

As outlined in the INTRODUCTION, we need sensors for robots (1) when sensing is the robot's mission; (2) to support the robot's functioning in the environment; and (3) for proprioception, i.e., robot 'self awareness'.

What sensors are required for the mission cannot be answered specifically until the mission is specified. But we can say quite confidently that the answers will relate to instrumentation: thermometers, hygrometers, barometers, densitometers, spectrometers, light meters, tilt meters, gravity meters, and so on. Many of these are already available as microsensors ${ }^{3}$; most of the rest are already available as minisensors. Some that at first glance seem ultimately resistant to shrinking are understood with more careful examination to be fundamentally improved if make smaller, e.g., mass spectrometers.

The sensors needed for the robot to function in the mission environment generally relate to navigation and to danger; the two are, of course, often closely linked! Exactly what sensors are required depends on the nature of the environment, its predictability, and the extent to which the mission can accept the risk of not sensing something because it is a priori believed to be uninteresting. These sorts of sensors are usually also important to the automobile industry, putting robotics in the unusually pleasant position of benefiting from the mass market's pressure to make them (and also communication devices) cheaper, smaller, and better. The common requirements include temperature, pressure, sound, moisture, acceleration and angular rate, chemistry, vision, radar and sonar, externally referenced navigation, e.g., GPS, among others.

The sensors needed for proprioception are largely a subset of those needed for the robot to function in the environment, with the addition of a few such as joint angle, strain, tilt, vibration, etc. The proprioceptive modality similarly benefits from the emergence of automobiles as sensor-intensive, selfdiagnosing machines.

Finally, do they need to be 'smart sensors'? It depends partly on what we mean by a 'smart sensor'. Of course, some need to be 'smart' no matter how we define it: sensors that compress large volumes of low value data into small volumes of high value information, for example, vision systems and radars, obviously fit better into a control system if they internalize most of the computing power they require for digital signal processing and high level reasoning. Sensors for even simple measurands like temperature and pressure may similarly benefit from local intelligence if the conclusion they generate is abstracted from the integration of many such simple sensors. On the other hand, if all the system needs to know is that, say, one critical temperature is below a particular limit, it is hard to justify making that sensor smarter than and analog-to-digital converter.

\section{REFERENCES}

[1] Noser, Stefan, seminar "Natürliche und Künstliche Intelligenz", lecture "Foundations of Classical AI and Cognitive Science", March 2001, Institut fur Informatik der Universitat Zurich. See http://www.ifi.unizh.ch/groups/ailab/teaching/semi2000/semi.htm.

[2] Malcolm, Chris, The Ignorance of Springs and Levers, http://www.dai.ed.ac.uk/homes/cam/drafts/cog/node10.html.

[3] Capek, Karel, (translated by P. Selver, and N. R. Playfair), R. U. R. (Rossum's universal robots) : a fantastic melodrama in three acts and an epilogue. New York: S. French, 1923.

[4] Morita, Akio, From a 500-dollar Company to a Global Corporation: The Growth of Sony, Carnegie Mellon University Press, Pittsburgh, 1985.

[5] Searches using http://www.google.com/ in February and March 2001.

[6] Siegel, M., P. Gunatilake, and G. Podnar, Robotic Assistants for Aircraft Inspectors, Instrumentation and Measurement Magazine, Vol. 1, No. 1, March, 1998, pp. 16 - 30. Also see CIMP project website http://www.ri.cmu.edu/projects/project_85.html

[7] Nourbakhsh, Illah, http://www.cs.cmu.edu/ pprk/index.html

[8] Allumette "microrobot" family portrait (unfortunately little text) at http://www.ifr.mavt.ethz.ch/photo/mobile/

[9] Baur, C., B. C. Gazen, B. Koel, T. R. Ramachandran, A. A. G. Requicha, and L. Zini, "Robotic Nanomanipulation with an SPM in a Networked Computing Environment", at http://wwwpal.usc.edu/ 1mr/publications/NanoIV/.

[10] Dual, J., and R. Buser, "Polyproject Nanorobot", at http://www.ifm.mavt.ethz.ch/research/nano-2.html.

[11] Weiss, Ron, MIT AI Lab, see http://www.swiss.ai.mit.edu/ rweiss/bioprogramming/.

[12] Feynmann, Richard P., in California Institute of Technology's Engineering and Science magazine, available on the web at http://www.zyvex.com/nanotech/feynman.htm

[13] For example, Faulhaber MicroMo Motors, http:Ilwww.micromo.com, Brushless DC Motor 0206, $1.9 \mathrm{~mm}$ diameter by $6 \mathrm{~mm}$ length. Details at http://209.207.212.142/detail.asp?Pk_product_id=207.

[14] Sandia Laboratories, "Dynamometer" web page at http://www.mdl.sandia.gov/micromachine/images11.html.

[15] Sandia Laboratories "Bugs on Devices" web page at http://www.mdl.sandia.gov/micromachine/images.html.

[16] Yeh, Richard, "photo gallery" web page at http://wwwbsac.eecs.berkeley.edu/ yeh/sems.html.

[17] Austin, Ian, "The Incredible Shrinking Robot, Self Contained and Untethered", New York Times, "What's Next" column, Feb 22, 2001. Also http://www.sandia.gov/media/NewsRel/NR2001/minirobot.htm.

[18] See description of MARV microrobot at http://www.sandia.gov/isrc/Capabilities/Prototyping/Small_Smart_Machine s/MARV/marv.html.

[19] http://dmtwww.epfl.ch/ cglauser/AliceGallery/Gallery.html Alice "family" website, see http://dmtwww.epfl.ch/ cglauser/AliceGallery/Gallery.html.

[20] EPFL Alice ' 99 website, see http://dmtwww.epfl.ch/isr/asl/projects/alice_pj.html.

[21] BoeBot web page at http://www.parallaxinc.com/.

[22] Runnabout robot manufactured by Silicon Sound, described at http://popularmechanics.com/popmech/sci/tech/9601TURBVM.html.

[23] Descartes robot manufactured by Diversified Enterprises, described at http://www.divent.com/.

[24] Khepera robot designed at EPFL, described at http://diwww.epfl.ch/Khepera/ - khepera.

[25] SuBot robot designed by SAIC, described at http://www.csee.usf.edu/robotics/Equipment/saic.html.

[26] SubMar underwater robot from HUT ATL described at http://www.automation.hut.fi/research/robotics/society/main.htm.

\footnotetext{
${ }^{3}$ Using the same prefix - size relationship as we adopted for robots.
} 